\title{
One dimensional combination technique and its implementation
}

\author{
Y. Fang $^{1}$
}

(Received 29 January 2011; revised 15 July 2011)

\begin{abstract}
This article introduces the $1 \mathrm{D}$ combination technique and its implementation with parallel programming. I discuss two primary features of the 1D combination technique: (1) its reduction of computational cost, especially when combined with parallel programming and where high accuracy is required; and (2) a resultant sacrifice of accuracy. However, the loss of the accuracy can be bounded thus reducing its significance.
\end{abstract}

\section{Contents}

1 Introduction

C645

2 Preliminaries

C646

http://anziamj.austms.org.au/ojs/index.php/ANZIAMJ/article/view/3935 gives this article, (C) Austral. Mathematical Soc. 2011. Published August 10, 2011. ISSN 1446-8735. (Print two pages per sheet of paper.) Copies of this article must not be made otherwise available on the internet; instead link directly to this URL for this article. 
3 One dimensional combination technique $\quad$ C648

3.1 Proof of Theorem 4 . . . . . . . . . . . . C648

3.2 Numerical experiments . . . . . . . . . . . . . . . C655

4 Parallel complexity of $1 \mathrm{D}$ combination technique

C655

5 Conclusion

C657

References

C659

\section{Introduction}

The discretization of PDEs by standard finite element approaches is limited to problems with up to three or four dimensions, due to the curse of dimensionality. Zenger and others [1,2] introduced sparse grid approximations and the combination technique, which substantially reduces computational complexity at a moderate cost in accuracy, allowing the numerical treatment of problems with ten variables or more and making the numerical solution of finite element methods feasible on computational equipment currently available.

My intention is to introduce new parallel implementation techniques to increase the parallelism and reduce the parallel complexity in computation for high dimensional problems. I study the one dimensional problem as a model problem to illustrate these new parallel implementation techniques which are applicable to higher dimensional cases. A one dimensional parallel implementation technique is suggested here. I prove that in one dimension the full grid approximation can be replaced by a linear combination of certain partial fine grid approximations with a bounded error. The parallel speedup is also discussed. 


\section{Preliminaries}

We study the following variational formulation of an elliptic boundary value problem: find $u \in \mathbf{H}^{1}([0,1])$ such that

$$
\mathrm{a}(\mathrm{u}, v)=(\mathrm{f}, v) \text { for all } v \in \mathbf{H}^{1}([0,1]),
$$

where the bilinear form $\mathbf{a}(\cdot, \cdot)$ is continuous and coercive in $\mathbf{H}^{1}([0,1])$. Here $\mathbf{H}^{1}([0,1])$ is the Sobolev space $\mathbf{W}_{2}^{1}([0,1])$ of functions with both their first derivatives and themselves in $\mathbf{L}^{2}([0,1])$. Assume that $a(\cdot, \cdot)$ takes the form $a(u, v)=\int_{0}^{1}\left(a_{1} u^{\prime} v^{\prime}+a_{2} u^{\prime} v+a_{3} u v\right)$ where $(\cdot, \cdot)$ denotes the $\mathbf{L}^{2}$-inner product and $f \in \mathbf{L}^{2}([0,1])$.

To find the Ritz-Galerkin projection of the solution of (1) in some finite dimensional subspace of $\mathbf{H}^{1}([0,1])$, we introduce piecewise linear function spaces. Let $L \in \mathbb{N} \cup\{0\}$ and $h_{\mathrm{L}}:=2^{-\mathrm{L}}$. We define a uniform partition $\mathfrak{R}_{\mathrm{L}}$, with level $L$ of the region $\Omega$, as a set of subintervals with width $h_{L}$ such that the union of these subintervals is $\Omega$ and such that the intersection of two subintervals of $\mathfrak{R}_{\mathrm{L}}$ either consists of a common vertex of both subintervals or is empty. Piecewise linear nodal hat functions $\phi_{\mathrm{L}, i}(i$ indicates the $i$ th nodal point) form a basis of the approximation space $\mathbf{V}_{\mathrm{L}} \subset \mathbf{H}^{1}([0,1])$ corresponding to the grid $\Omega_{\mathrm{L}}$. The Ritz-Galerkin projection of the solution of (1) into the space $V_{L}$ is the solution $\mathfrak{u}_{\mathrm{L}} \in \mathrm{V}_{\mathrm{L}}$ of

$$
\mathrm{a}\left(\mathrm{u}_{\mathrm{L}}, v_{\mathrm{L}}\right)=\left(\mathrm{f}, v_{\mathrm{L}}\right) \quad \text { for all } v_{\mathrm{L}} \in \mathrm{V}_{\mathrm{L}} \text {. }
$$

This equation leads to a linear system $M c=b$ where $M_{i j}:=a\left(\phi_{L, j}, \phi_{L, i}\right)$ and $b_{i}:=\left(f, \phi_{\mathrm{L}, i}\right)$. This is termed as the full grid Galerkin method.

Before introducing the 1D combination technique, we introduce the following notation.

1. Let $\mathfrak{R}_{k,-}$ be a partition with level $k \in \mathbb{N} \cup\{0\}$ of $[0,1 / 2]$. Denote the resulting grid by $\Omega_{k,-}$ and the corresponding piecewise linear function space by $V_{k,-}$. Thus, a function $v \in \mathrm{H}^{1}([0,1])$ belongs to $\mathrm{V}_{\mathrm{k},-}$ if and only if $\left.v\right|_{[0,1 / 2]}$ belongs to the span of $\left.\phi_{k+1, i}\right|_{[0,1 / 2]}$ for $i=0,1, \ldots, 2^{k}$. 
2. Let $\mathfrak{R}_{-, l}$ be a partition with level $l \in \mathbb{N} \cup\{0\}$ of $[1 / 2,1]$. Denote the resulting grid by $\Omega_{-, l}$ and the corresponding piecewise linear function space by $\mathrm{V}_{-, \mathrm{l}}$. Thus, a function $v \in \mathrm{H}^{1}([0,1])$ belongs to $\mathrm{V}_{-, \mathrm{l}}$ if and only if $\left.v\right|_{[1 / 2,1]}$ belongs to the span of $\left.\phi_{l+1, i}\right|_{[1 / 2,1]}$ for $i=2^{l}, 2^{l}+1, \ldots, 2^{l+1}$.

3. Let $\mathfrak{R}_{\mathrm{k}, \mathrm{l}}$ be a partition with level $k \in \mathbb{N} \cup\{0\}$ of $[0,1 / 2]$ and level $l \in \mathbb{N} \cup$ $\{0\}$ of $[1 / 2,1]$. Denote the resulting grid by $\Omega_{k, l}$ and the corresponding piecewise linear function space by $\mathrm{V}_{k, l}$. Thus, a function $v \in H^{1}([0,1])$ belongs to $V_{k, l}$ if and only if $\left.v\right|_{[0,1 / 2]}$ belongs to the span of $\left.\phi_{k+1, i}\right|_{[0,1 / 2]}$ for $i=0,1, \ldots, 2^{k}$ and $\left.v\right|_{[1 / 2,1]}$ belongs to the span of $\left.\phi_{l+1, i}\right|_{[1 / 2,1]}$ for $i=2^{l}, 2^{l}+1, \ldots, 2^{l+1}$.

Let $P_{\mathrm{L}} \mathfrak{u}, \mathrm{P}_{k,-} \mathfrak{u}, \mathrm{P}_{-,,} \mathfrak{u}, \mathrm{P}_{k, l} \mathfrak{u}$ and $\mathrm{I}_{\mathrm{L}} \mathfrak{u}, \mathrm{I}_{k,-} \mathfrak{u}, \mathrm{I}_{-, l} \mathfrak{u}, \mathrm{I}_{k, l} \mathfrak{u}$ denote the Galerkin projections and the interpolants of the solution $\boldsymbol{u}$ of (1) into the spaces $\mathbf{V}_{\mathrm{L}}$, $\mathbf{V}_{\mathrm{k},-}, \mathbf{V}_{-, l}, \mathbf{V}_{\mathrm{k}, \mathrm{l}}$, respectively. $\mathbf{V}_{\mathrm{n}, \mathrm{n}}=\mathbf{V}_{\mathrm{n}+1}$. By the uniqueness of the Galerkin projection and the interpolant, for any $\mathbf{u} \in \mathbf{H}^{1}([0,1])$,

$$
\begin{aligned}
& P_{n, n} u(x)=P_{n+1} u(x), \quad n \in \mathbb{N} \cup\{0\}, \\
& I_{n, n} u(x)=I_{n+1} u(x), \quad n \in \mathbb{N} \cup\{0\}, \\
& I_{k, l} u(x)=\left(I_{k,-} \circ I_{-, l}\right) u(x), \quad n \in \mathbb{N} \cup\{0\} .
\end{aligned}
$$

For notational convenience, the symbols $\lesssim, \gtrsim$ and $\approx$ are used in this article. The expressions $x_{1} \lesssim y_{1}, x_{2} \gtrsim y_{2}$ and $x_{3} \approx y_{3}$ mean that $x_{1} \leqslant c_{1} y_{1}$, $x_{2} \geqslant C_{2} y_{2}$ and $c_{3} y_{3} \leqslant x_{3} \leqslant C_{3} y_{3}$ for some strictly positive constants $C_{1}$, $\mathrm{C}_{2}, \mathrm{C}_{3}$, and $\mathrm{c}_{3}$ that are independent of mesh parameters. The following two lemmas from Pflaum and Zhou [3] are used later.

Lemma 1 ([3, Lemma 2]). Let $\mathrm{L} \in \mathrm{N} \cup\{0\}$ and $\mathrm{h}=2^{-\mathrm{L}}$. For $\mathrm{e}=\left[\mathrm{x}_{\mathrm{e}}-\right.$ $\left.h / 2, x_{e}+h / 2\right] \in \Omega_{L}$,

$$
\int_{e} w-\mathrm{I}_{\mathrm{L}} w=\int_{e} \mathrm{E}_{e} w^{\prime \prime}, \quad \text { where } \mathrm{E}_{e}(\mathrm{x})=\frac{1}{2}\left(\mathrm{x}-\mathrm{x}_{e}\right)^{2}-\frac{1}{8} \mathrm{~h}^{2} .
$$

Lemma 2 ([3, Lemma 3(ii)]). Assume $\boldsymbol{w} \in \mathbf{H}^{3}([0,1])$. Let $\mathbf{L} \in \mathbb{N} \cup\{0\}$ and $\mathrm{h}=2^{-\mathrm{L}}$ be the mesh size of the uniform grid $\Omega_{\mathrm{L}}$ on $[0,1]$. If $\phi, \phi^{\prime} \in$ 
$\mathbf{L}^{\infty}([0,1])$, then there exists $\mathrm{f}^{1}, \mathrm{f}^{2} \in \mathbf{V}_{\mathrm{L}}$ such that

$$
\begin{aligned}
& \int_{0}^{1} \phi\left(w-\mathrm{I}_{\mathrm{L}} w\right) v^{\prime}=\left(\mathrm{f}^{1}, v\right) \quad \text { for all } v \in \mathrm{V}_{\mathrm{L}}, \quad \text { with }\left\|\mathrm{f}^{1}\right\|_{\mathrm{L}^{2}} \lesssim \mathrm{h}^{2}\|w\|_{\mathbf{H}^{3}}, \\
& \int_{0}^{1} \phi\left(w-\mathrm{I}_{\mathrm{L}} w\right)^{\prime} v=\left(\mathrm{f}^{2}, v\right) \quad \text { for all } v \in \mathrm{V}_{\mathrm{L}}, \quad \text { with }\left\|\mathrm{f}^{2}\right\|_{\mathrm{L}^{2}} \lesssim h^{2}\|w\|_{\mathbf{H}^{3}} .
\end{aligned}
$$

\section{One dimensional combination technique}

Definition 3.

$$
\mathfrak{u}_{n}^{\mathfrak{c}}:=\sum_{k+l=n} P_{k, l} \mathfrak{u}-\sum_{k+l=n-1} P_{k, l} \mathfrak{u} \text { where } n \geqslant 1 .
$$

Theorem 4. Assume $\mathbf{u} \in \mathbf{H}^{3}([0,1]), \mathrm{a}_{1} \in \mathbf{W}_{\infty}^{1}([0,1])$ and $\mathbf{a}_{2}, \mathrm{a}_{3} \in \mathbf{L}^{\infty}([0,1])$ where $\mathrm{a}_{1}, \mathrm{a}_{2}$ and $\mathrm{a}_{3}$ are the coefficients in the bilinear form $\mathbf{a}(\mathbf{u}, v)$. Then for $\mathrm{n} \in \mathbf{N}$,

$$
\left\|P_{n, n} u-u_{n}^{c}\right\|_{\mathbf{L}^{2}} \lesssim h_{n}^{2} \log _{2}\left(h_{n}^{-1}\right)\|u\|_{\mathbf{H}^{3}}, \quad\left\|P_{n, n} u-u_{n}^{c}\right\|_{\mathbf{H}^{1}} \lesssim h_{n}\|u\|_{\mathbf{H}^{3}} .
$$

\subsection{Proof of Theorem 4}

Introduce the indices $\alpha, \beta \in\{0,1\}^{2}$ with the norm $|\alpha|=\alpha_{1}+\alpha_{2}$, and let $\mathbf{0}=(0,0)$ and $\mathbf{e}=(1,1)$. In the context of the $2 \mathrm{D}$ index, component-wise arithmetic operations are used.

Let $P_{k, l}$ and $I_{k, l}$ be a sequence of the Ritz-Galerkin projection operators and a sequence of the interpolation operators from $\mathbf{H}^{1}([0,1])$ into the space $\mathbf{V}_{k, l}$, respectively. Let $\mathrm{F}_{\mathrm{k}, \mathrm{l}}$ denote either $\mathrm{P}_{\mathrm{k}, \mathrm{l}}$ or $\mathrm{I}_{\mathrm{k}, \mathrm{l}}$. The hierarchical surplus operator $\delta^{\mathbf{e}}$ is defined by Pflaum and Zhou [3] as

$$
\delta^{\mathbf{e}} \mathrm{F}_{\mathrm{k}, \mathrm{l}} \mathrm{w}:=(-1)^{|\mathrm{e}|} \cdot\left[\sum_{0 \leqslant \beta \leqslant \mathrm{e}}(-1)^{|\beta|} \mathrm{F}_{k+\beta_{1}, l+\beta_{2}} w\right] .
$$


Lemma 5 (Error decomposition form).

$$
P_{n, n} u-u_{n}^{c}=\sum_{k=0}^{n-1} \sum_{l=n-k-1}^{n-1} \delta^{e} P_{k, l} u, \quad n \geqslant 1 .
$$

Proof: Let $m \in \mathbb{Z}$ and $0 \leqslant m \leqslant n-1$, based on the definition of the hierarchical surplus operator $\delta^{\mathbf{e}}$ (equation (5)), we have

$$
\begin{aligned}
\sum_{k=m}^{n-1} \delta^{\mathbf{e}} P_{k, n-1-k+m} u= & \sum_{k=m}^{n-1} P_{k+1, n-k+m} u-\sum_{k=m}^{n-1} P_{k+1, n-1-k+m} u \\
& -\sum_{k=m}^{n-1} P_{k, n-k+m} u+\sum_{k=m}^{n-1} P_{k, n-1-k+m} u \\
= & \sum_{k=m+1}^{n} P_{k, n-k+m+1} u-\sum_{k=m+1}^{n} P_{k, n-k+m} u \\
& -\sum_{k=m}^{n-1} P_{k, n-k+m} u+\sum_{k=m}^{n-1} P_{k, n-1-k+m} u \\
= & \sum_{k=m+1}^{n-1} P_{k, n-k+m+1} u-\sum_{k=m+1}^{n-1} P_{k, n-k+m} u \\
& -\sum_{k=m}^{n-1} P_{k, n-k+m} u+\sum_{k=m}^{n-1} P_{k, n-1-k+m} u \\
& +P_{n, m+1} u-P_{n, m} u .
\end{aligned}
$$

Summing $\mathrm{m}$ from 0 to $\mathrm{n}-1$,

$$
\begin{aligned}
& \sum_{m=0}^{n-1} \sum_{k=m}^{n-1} \delta^{e} P_{k, n-1-k+m} u \\
= & {\left[\sum_{k=m+1}^{n} P_{k, n-k+m+1} u-\sum_{k=m+1}^{n} P_{k, n-k+m} u\right]_{m=n-1} }
\end{aligned}
$$




$$
\begin{aligned}
& -\left[\sum_{k=m}^{n-1} P_{k, n-k+m} u+\sum_{k=m}^{n-1} P_{k, n-1-k+m} u\right]_{m=0}+\sum_{m=0}^{n-2}\left(P_{n, m+1} u-P_{n, m} u\right) \\
= & P_{n, n} u-\sum_{k=0}^{n} P_{k, n-k} u+\sum_{k=0}^{n-1} P_{k, n-1-k} u .
\end{aligned}
$$

On the other hand,

$$
\sum_{m=0}^{n-1} \sum_{k=m}^{n-1} \delta^{\mathbf{e}} P_{k, n-1-k+m} u=\sum_{k=0}^{n-1} \sum_{l=n-k-1}^{n-1} \delta^{e} P_{k, l} u .
$$

Thus, by the definition (3) of $\mathfrak{u}_{\mathfrak{n}}^{\mathfrak{c}}$, we finish the proof.

Lemma 6. Assume $\mathrm{u} \in \mathbf{H}^{3}([0,1])$. If $\mathrm{a}_{1}, \mathrm{a}_{2} \in \mathbf{W}_{\infty}^{1}([0,1])$ and $\mathrm{a}_{3} \in$ $\mathbf{L}^{\infty}([0,1])$, then there exists $\boldsymbol{w} \in \mathbf{H}^{2}([0,1])$ such that

$$
\mathrm{P}_{\mathrm{k}, \mathrm{l}}\left(\mathrm{I}-\mathrm{I}_{\mathrm{k},-}\right) \mathrm{u}=\mathrm{P}_{\mathrm{k}, \mathrm{l}} \mathrm{w}, \quad \text { with }\|w\|_{\mathbf{H}^{2}} \lesssim \mathrm{h}_{\mathrm{k}+1}^{2}\|\mathrm{u}\|_{\mathbf{H}^{3}} .
$$

Similarly, there exists $v \in \mathbf{H}^{2}([0,1])$ such that

$$
\mathrm{P}_{\mathrm{k}, \mathrm{l}}\left(\mathrm{I}-\mathrm{I}_{-, \mathrm{l}}\right) \mathrm{u}=\mathrm{P}_{\mathrm{k}, \mathrm{l}} \mathrm{v}, \quad \text { with }\|v\|_{\mathbf{H}^{2}} \lesssim \mathrm{h}_{\mathrm{l+1}}^{2}\|\mathrm{u}\|_{\mathbf{H}^{3}} .
$$

Proof: For any $v_{\mathrm{k}, \mathrm{l}} \in \mathbf{V}_{\mathrm{k}, \mathrm{l}}$,

$$
\begin{aligned}
a\left(\left(I-I_{k,-}\right) u, v_{k, l}\right)= & \int_{0}^{1} a_{1}\left[\left(I-I_{k,-}\right) u\right]^{\prime} v_{k, l}^{\prime}+a_{2}\left[\left(I-I_{k,-}\right) u\right]^{\prime} v_{k, l} \\
& +a_{3}\left(I-I_{k,-}\right) u v_{k, l} .
\end{aligned}
$$

Let $T_{k, l}$ be the set of all non-overlapping mesh intervals from $\Omega_{k, l}$,

$$
\int_{0}^{1} a_{1}\left[\left(I-I_{k,-}\right) u\right]^{\prime} v_{k, l}^{\prime}=\sum_{e \in T_{k, l}} \int_{e} a_{1}\left[\left(I-I_{k,-}\right) u\right]^{\prime} v_{k, l}^{\prime},
$$


where for each $e$, when $y \in \partial e$,

$$
v_{k, l}^{\prime}(y):=\lim _{x \rightarrow y, x \in e} \frac{v_{k, l}(x)-v_{k, l}(y)}{x-y} .
$$

Since $u \in \mathbf{H}^{3}([0,1])$, ( $\left.\mathrm{I}-\mathrm{I}_{\mathrm{k},-}\right) \mathrm{u}$ is sufficiently smooth on $e$, so

$$
\lim _{x \rightarrow y, x \in e}\left(I-I_{k,-}\right) u(x)=\left(I-I_{k,-}\right) u(y)=0,
$$

and thus for any $y \in \partial e$,

$$
\left(a_{1}\left(I-I_{k,-}\right) u v_{k, l}^{\prime}\right)(y)=\lim _{x \rightarrow y, x \in e}\left(a_{1}\left(I-I_{k,-}\right) u v_{k, l}^{\prime}\right)(x)=0 .
$$

Now on each $e$, we look at $a_{1} \cdot v_{k, l}^{\prime}$ as one function and $\left(I-I_{k,-}\right) u$ as another function. By integration by parts,

$$
\begin{aligned}
\int_{e} a_{1} \cdot\left[\left(I-I_{k,-}\right) u\right]^{\prime} v_{k, l}^{\prime}= & \left.a_{1}\left(I-I_{k,-}\right) u v_{k, l}^{\prime}\right|_{\partial e}-\int_{e} a_{1}^{\prime}\left(I-I_{k,-}\right) u v_{k, l}^{\prime} \\
& -\int_{e} a_{1}\left(I-I_{k,-}\right) u v_{k, l}^{\prime \prime} .
\end{aligned}
$$

The equation (6) tells us the term $\left.a_{1}\left(I-I_{k,-}\right) \mathfrak{u} v_{k, l}^{\prime}\right|_{\partial e}=0$. Moreover, since $v_{k, l} \in \mathbf{V}_{k, l}$ is piecewise linear on $e, v_{k, l}^{\prime \prime}=0$. Thus

$$
\int_{e} a_{1} \cdot\left[\left(I-I_{k,-}\right) u\right]^{\prime} v_{k, l}^{\prime}=-\int_{e} a_{1}^{\prime}\left(I-I_{k,-}\right) u v_{k, l}^{\prime} .
$$

After summing up all $e \in T_{k, l}$,

$\int_{0}^{1} a_{1} \cdot\left[\left(I-I_{k,-}\right) u\right]^{\prime} v_{k, l}^{\prime}=\sum_{e \in T_{k, l}}-\int_{e} a_{1}^{\prime}\left(I-I_{k,-}\right) u v_{k, l}^{\prime}=-\int_{0}^{1} a_{1}^{\prime}\left(I-I_{k,-}\right) u v_{k, l}^{\prime}$.

Furthermore,

$$
a\left(\left(I-I_{k,-}\right) u, v_{k, l}\right)=\int_{0}^{1}-a_{1}^{\prime}\left(I-I_{k,-}\right) u v_{k, l}^{\prime}+a_{2}\left[\left(I-I_{k,-}\right) u\right]^{\prime} v_{k, l}
$$




$$
+\mathrm{a}_{3}\left(\mathrm{I}-\mathrm{I}_{\mathrm{k},-}\right) \mathrm{u} v_{\mathrm{k}, \mathrm{l}} .
$$

Since $a_{1}, a_{2} \in \mathbf{W}_{\infty}^{1}([0,1])$, by Lemma 2 , there exist $g^{1}, g^{2} \in \mathbf{V}_{k, l}$ with $\left.\left.\mathrm{g}^{1}\right|_{\left[\frac{1}{2}, 1\right]} \equiv \mathrm{g}^{2}\right|_{\left[\frac{1}{2}, 1\right]} \equiv 0$, such that, for all $v \in \mathbf{V}_{\mathrm{k}, \mathrm{l}}$ with $\left\|\mathrm{g}^{1}\right\|_{\mathbf{L}^{2}} \lesssim \mathrm{h}_{\mathrm{k}+1}^{2}\|\mathrm{u}\|_{\mathbf{H}^{3}}$,

$$
\begin{aligned}
& \int_{0}^{1}-a_{1}^{\prime}\left(I-I_{k,-}\right) u v_{k, l}^{\prime}=\left(g^{1}, v_{k, l}\right) \\
& \int_{0}^{1} a_{2}\left[\left(I-I_{k,-}\right) u\right]^{\prime} v_{k, l}=\left(g^{2}, v_{k, l}\right)
\end{aligned}
$$

As $a_{3} \in \mathbf{L}^{\infty}([0,1])$,

$$
\left\|a_{3}\left(I-I_{k,-}\right) u\right\|_{L^{2}} \lesssim\left\|\left(I-I_{k,-}\right) u\right\|_{L^{2}} \leqslant h_{k+1}^{2}\|u\|_{\mathbf{H}^{2}} .
$$

Hence, there exists a function $g \in \mathbf{L}^{2}([0,1])$ such that $\mathrm{a}\left(\left(\mathrm{I}-\mathrm{I}_{\mathrm{k},-}\right) \mathrm{u}, v_{\mathrm{k}, \mathrm{l}}\right)=\left(\mathrm{g}, v_{\mathrm{k}, \mathrm{l}}\right), \quad$ for all $v \in \mathrm{V}_{\mathrm{k}, \mathrm{l}}$ with $\|\mathrm{g}\|_{\mathrm{L}^{2}} \lesssim \mathrm{h}_{\mathrm{k}+1}^{2}\|\mathrm{u}\|_{\mathbf{H}^{3}}$. On the other hand, by the Lax-Milgram theorem, there exists $\boldsymbol{w} \in \mathbf{H}^{2}([0,1])$ satisfying

$$
\mathrm{a}(\boldsymbol{w}, v)=(\mathrm{g}, v), \quad \text { for all } v \in \mathbf{H}^{1}([0,1]) \quad \text { with }\|w\|_{\mathbf{H}^{2}} \lesssim\|g\|_{\mathbf{L}^{2}} .
$$

Thus, $\mathrm{a}\left(\left(\mathrm{I}-\mathrm{I}_{\mathrm{k},-}\right) \mathrm{u}, v_{\mathrm{k}, \mathrm{l}}\right)=\mathrm{a}\left(\boldsymbol{w}, v_{\mathrm{k}, \mathrm{l}}\right)$ with $\|w\|_{\mathbf{H}^{2}} \lesssim \mathrm{h}_{\mathrm{k}+1}^{2}\|\mathrm{u}\|_{\mathbf{H}^{3}}$. The proof of the second part of Lemma 6 is similar and is omitted.

Lemma 7. Let $\boldsymbol{w} \in \mathbf{H}^{2}([0,1])$. Then

$$
\left\|\delta^{0,1} \mathrm{P}_{\mathrm{k}, \mathrm{l}} w\right\|_{\mathbf{L}^{2}} \lesssim h_{\mathrm{l}+1}^{2} \cdot\|w\|_{\mathbf{H}^{2}}, \quad\left\|\delta^{0,1} \mathrm{P}_{\mathrm{k}, \mathrm{l}} w\right\|_{\mathbf{H}^{1}} \lesssim h_{\mathrm{l}+1} \cdot\|w\|_{\mathbf{H}^{2}}
$$

and

$$
\left\|\delta^{1,0} \mathrm{P}_{\mathrm{k}, \mathrm{l}} w\right\|_{\mathbf{L}^{2}} \lesssim h_{\mathrm{k}+1}^{2} \cdot\|w\|_{\mathbf{H}^{2}}, \quad\left\|\delta^{1,0} \mathrm{P}_{\mathrm{k}, \mathrm{l}} \boldsymbol{w}\right\|_{\mathbf{H}^{1}} \lesssim h_{\mathrm{k}+1} \cdot\|w\|_{\mathbf{H}^{2}}
$$


Proof: Let $\mathbf{g} \in \mathbf{V}_{\mathrm{k},-}$ be the solution of

$$
\mathrm{a}(v, \mathrm{~g})=\int_{\Omega} v \cdot\left(\mathrm{P}_{\mathrm{k},-}-\mathrm{P}_{\mathrm{k}, \mathrm{l}}\right) w, \quad \text { for all } v \in \mathrm{V}_{\mathrm{k},-} .
$$

By observing $\mathrm{I}_{-, \mathrm{l}} \mathrm{g} \in \mathbf{V}_{\mathrm{k}, \mathrm{l}}$,

$$
\begin{aligned}
\left\|\left(P_{k,-}-P_{k, l}\right) w\right\|_{L^{2}}^{2} & =a\left(\left(P_{k,-}-P_{k, l}\right) w, g\right) \\
& =a\left(\left(P_{k,-}-P_{k, l}\right) w, g-I_{-, l} g\right) \\
& \lesssim\left\|\left(P_{k,-}-P_{k, l}\right) w\right\|_{\mathbf{H}^{1}}\left\|g-I_{-, l} g\right\|_{\mathbf{H}^{1}} \\
& \lesssim\left\|\left(P_{k,-}-P_{k, l}\right) w\right\|_{\mathbf{H}^{1}} 2^{-l-1}\left\|g^{\prime}\right\|_{\mathbf{H}^{1}} .
\end{aligned}
$$

By elliptic regularity, $\|g\|_{\mathbf{H}^{2}} \lesssim\left\|\left(\mathrm{P}_{\mathrm{k},-}-\mathrm{P}_{\mathrm{k}, \mathrm{l}}\right) \mathcal{w}\right\|_{\mathbf{L}^{2}}$, so

$$
\left\|\left(\mathrm{P}_{\mathrm{k},-}-\mathrm{P}_{\mathrm{k}, \mathrm{l}}\right) w\right\|_{\mathrm{L}^{2}} \lesssim\left\|\left(\mathrm{P}_{\mathrm{k},-}-\mathrm{P}_{\mathrm{k}, \mathrm{l}}\right) w\right\|_{\mathbf{H}^{1}} \cdot 2^{-l-1}
$$

Since

$$
\begin{aligned}
\left\|\left(\mathrm{P}_{\mathrm{k},-}-\mathrm{P}_{\mathrm{k}, \mathrm{l}}\right) w\right\|_{\mathbf{H}^{1}} & =\left\|\left(\mathrm{P}_{\mathrm{k},-}-\mathrm{P}_{\mathrm{k}, \mathrm{l}} \mathrm{P}_{\mathrm{k},-}\right) w\right\|_{\mathbf{H}^{1}} \\
& \leqslant\left\|\left(\mathrm{I}-\mathrm{I}_{\mathrm{k}, \mathrm{l}}\right) \mathrm{P}_{\mathrm{k},-} w\right\|_{\mathbf{H}^{1}} \\
& \lesssim 2^{-\mathrm{l}-1}\|w\|_{\mathbf{H}^{2}},
\end{aligned}
$$

we obtain $\left\|\left(\mathrm{P}_{\mathrm{k},-}-\mathrm{P}_{\mathrm{k}, \mathrm{l}}\right) w\right\|_{\mathbf{L}^{2}} \lesssim 4^{-l-1}\|w\|_{\mathbf{H}^{2}}$. By the inequality

$$
\left\|\delta^{0,1} P_{k, l} w\right\| \leqslant\left\|\left(P_{k, l+1}-P_{k,-}\right) w\right\|+\left\|\left(P_{k,-}-P_{k, l}\right) w\right\|,
$$

the proof of the first part of Lemma 7 is finished. The proof of the second part is similar and is omitted.

Lemma 8. Assume $u \in \mathbf{H}^{3}([0,1])$. If $\mathrm{a}_{1} \in \mathbf{W}_{\infty}^{1}([0,1])$ and $\mathrm{a}_{2}, \mathrm{a}_{3} \in$ $\mathbf{L}^{\infty}([0,1])$, then

$$
\begin{aligned}
& \left\|\delta^{\mathbf{e}} \mathrm{P}_{k, l} u\right\|_{\mathbf{L}^{2}} \\
& \left\|h_{k+1}^{2} h_{l+1}^{2}\right\| u \|_{\mathbf{H}^{3}} \\
& \left\|\delta^{\mathbf{e}} P_{k, l} u\right\|_{\mathbf{H}^{1}} \lesssim h_{k+1} h_{l+1}\left(h_{k+1}+h_{l+1}\right)\|u\|_{\mathbf{H}^{3}} .
\end{aligned}
$$


Proof: We note that

$$
\begin{aligned}
\delta^{\mathbf{e}} \mathrm{P}_{k, l} \mathrm{u} & =\delta^{\mathbf{e}}\left(\mathrm{P}_{k, l}-\mathrm{P}_{k, l} \mathrm{I}_{k, l}\right) u+\delta^{\mathbf{e}} \mathrm{P}_{k, l} \mathrm{I}_{k, l} \mathrm{u} \\
& =\delta^{\mathbf{e}} \mathrm{P}_{k, l}\left(\mathrm{I}-\mathrm{I}_{k, l}\right) \mathrm{u}+\delta^{\mathbf{e}} \mathrm{I}_{k, l} \mathrm{u} .
\end{aligned}
$$

Since $\mathrm{I}_{k, \mathrm{l}}=\mathrm{I}_{k,-} \circ \mathrm{I}_{-, \mathrm{l}}$ and

$$
\begin{aligned}
\mathrm{I} & =\mathrm{I}_{\mathrm{k}, \mathrm{l}}+\left(\mathrm{I}-\mathrm{I}_{\mathrm{k},-}\right)+\left(\mathrm{I}-\mathrm{I}_{-, \mathrm{l}}\right)-\left(\mathrm{I}-\mathrm{I}_{\mathrm{k},-}\right)\left(\mathrm{I}-\mathrm{I}_{-, \mathrm{l}}\right) \\
& =\mathrm{I}_{\mathrm{k}, \mathrm{l}}+\left(\mathrm{I}-\mathrm{I}_{\mathrm{k},-}\right)+\left(\mathrm{I}-\mathrm{I}_{-, \mathrm{l}}\right),
\end{aligned}
$$

then

$$
\begin{aligned}
\delta^{\mathbf{e}} \mathrm{P}_{k, l} \mathrm{u} & =\delta^{\mathbf{e}} \mathrm{P}_{k, l}\left(\mathrm{I}-\mathrm{I}_{k,-}\right) \mathrm{u}+\delta^{\mathbf{e}} \mathrm{P}_{k, l}\left(\mathrm{I}-\mathrm{I}_{-, \mathrm{l}}\right) \mathrm{u}+\delta^{\mathbf{e}} \mathrm{I}_{k, l} \mathrm{u} \\
& =\delta^{1,0} \circ \delta^{0,1} \mathrm{P}_{k, l}\left(\mathrm{I}-\mathrm{I}_{\mathrm{k},-}\right) \mathrm{u}+\delta^{1,0} \circ \delta^{0,1} \mathrm{P}_{k, l}\left(\mathrm{I}-\mathrm{I}_{-, \mathrm{l}}\right) \mathrm{u}+\delta^{\mathbf{e}} \mathrm{I}_{k, l} \mathrm{u} .
\end{aligned}
$$

Let $\|\cdot\|$ be the norm $\|\cdot\|_{\mathbf{H}^{1}}$ or $\|\cdot\|_{\mathbf{L}^{2}}$. Then by the triangle inequality

$$
\begin{aligned}
\left\|\delta^{\mathbf{e}} \mathrm{P}_{k, l} \mathrm{u}\right\| \lesssim & \max _{\tilde{k}=k, k+1}\left\|\delta^{0,1} \mathrm{P}_{\tilde{\mathrm{k}}, \mathrm{l}}\left(\mathrm{I}-\mathrm{I}_{\tilde{\mathrm{k}},-}\right) \mathrm{u}\right\|+\max _{\tilde{\mathrm{l}}=l, l+1}\left\|\delta^{1,0} \mathrm{P}_{k, \tilde{l}}\left(\mathrm{I}-\mathrm{I}_{-, \tilde{l}}\right) \mathrm{u}\right\| \\
& +\left\|\delta^{\mathbf{e}} \mathrm{I}_{\mathrm{k}, \mathrm{l}} \mathrm{u}\right\|,
\end{aligned}
$$

where $\left\|\delta^{\mathbf{e}} I_{k, l} \mathfrak{u}\right\|=\left\|I_{k+1, l+1} \mathfrak{u}-\mathrm{I}_{k+1, l} \mathfrak{u}-\mathrm{I}_{k, l+1} \mathfrak{u}+\mathrm{I}_{k, l} \mathfrak{u}\right\|=0$. Furthermore, by Lemma 6 , there exist $\boldsymbol{w}_{1}, \boldsymbol{w}_{2} \in \mathbf{H}^{1}([0,1]) \cap \mathbf{H}^{2}([0,1])$ such that

$$
\begin{aligned}
\mathrm{P}_{\tilde{\mathrm{k}}, \mathrm{l}}\left(\mathrm{I}-\mathrm{I}_{\tilde{\mathrm{k}},-}\right) \mathrm{u}=\mathrm{P}_{\tilde{\mathrm{k}}, \mathrm{l}} \mathcal{w}_{1}, & \text { with }\left\|\boldsymbol{w}_{1}\right\|_{\mathbf{H}^{2}} \lesssim \mathrm{h}_{\tilde{\mathrm{k}}+1}^{2}\|\mathrm{u}\|_{\mathbf{H}^{3}}, \\
\mathrm{P}_{\mathrm{k}, \tilde{\mathrm{l}}}\left(\mathrm{I}-\mathrm{I}_{-, \tilde{l}}\right) \mathrm{u} & =\mathrm{P}_{\mathrm{k}, \tilde{\imath}} \mathcal{w}_{2}, \quad \text { with }\left\|\boldsymbol{w}_{2}\right\|_{\mathbf{H}^{2}} \lesssim \mathrm{h}_{\tilde{\mathrm{l}}+1}^{2}\|\mathrm{u}\|_{\mathbf{H}^{3}} .
\end{aligned}
$$

We have

$$
\left\|\delta^{\mathbf{e}} \mathrm{P}_{\mathrm{k}, \mathrm{l}} \mathrm{u}\right\| \lesssim \max _{\tilde{\mathrm{k}}=\mathrm{k}, \mathrm{k}+1}\left\|\delta^{0,1} \mathrm{P}_{\tilde{\mathrm{k}}, \mathrm{l}} \boldsymbol{w}_{1}\right\|+\max _{\tilde{\mathrm{l}}=\mathrm{l}, \mathrm{l}+1}\left\|\delta^{1,0} \mathrm{P}_{\mathrm{k}, \tilde{\mathrm{l}}} \boldsymbol{w}_{2}\right\|,
$$

so, by using Lemma 7 and Lemma 6,

$$
\left\|\delta^{\mathbf{e}} \mathbf{P}_{k, l} \mathfrak{u}\right\|_{\mathbf{L}^{2}} \lesssim \max _{\tilde{k}=k, k+1} h_{l+1}^{2}\left\|w_{1}\right\|_{\mathbf{H}^{2}}+\max _{\tilde{l}=l, l+1} h_{k+1}^{2}\left\|w_{2}\right\|_{\mathbf{H}^{2}}
$$




$$
\begin{aligned}
& \lesssim \max _{\tilde{k}=k, k+1} h_{l+1}^{2} h_{\tilde{k}+1}^{2}\|u\|_{\mathbf{H}^{3}}+\max _{\tilde{l}=l, l+1} h_{k+1}^{2} h_{\tilde{l}+1}^{2}\|u\|_{\mathbf{H}^{3}} \\
& \lesssim h_{k+1}^{2} h_{l+1}^{2}\|u\|_{\mathbf{H}^{3}},
\end{aligned}
$$

and

$$
\begin{aligned}
\left\|\delta^{\mathbf{e}} P_{k, l} u\right\|_{\mathbf{H}^{1}} & \lesssim \max _{\tilde{k}=k, k+1} h_{l+1}\left\|w_{1}\right\|_{\mathbf{H}^{2}}+\max _{\tilde{\mathrm{l}}=l, l+1} h_{k+1}\left\|w_{2}\right\|_{\mathbf{H}^{2}} \\
& \lesssim \max _{\tilde{k}=k, k+1} h_{\mathrm{l}+1} h_{\tilde{\mathrm{k}}+1}^{2}\|u\|_{\mathbf{H}^{3}}+\max _{\tilde{l}=l, l+1} h_{k+1} h_{\tilde{\mathrm{l}}+1}^{2}\|u\|_{\mathbf{H}^{3}} \\
& =h_{\mathrm{l}+1} h_{k+1}^{2}\|u\|_{\mathbf{H}^{3}}+h_{k+1} h_{\mathrm{l}+1}^{2}\|u\|_{\mathbf{H}^{3}} .
\end{aligned}
$$

Theorem 4 is a direct consequence of Lemma 5 and Lemma 8.

\subsection{Numerical experiments}

We report on numerical tests that support the 1D combination technique error estimate presented in Theorem 4 . We take the test problem $-\mathfrak{u}^{\prime \prime}(x)+$ $\mathfrak{u}^{\prime}(x)+\mathfrak{u}(x)=x, x \in[0,1]$ with $\mathfrak{u}^{\prime}(0)=\mathfrak{u}^{\prime}(1)=0$ in all the numerical experiments in this article. We note that the test problem ensures that the conditions of Theorem 4 have been satisfied. By comparing the last two columns of Table 1 and Table 2, one sees that the convergence rate of the error in the $\mathbf{L}^{2}$ norm is $\mathrm{O}\left(4^{-n} \mathbf{n}\right)$, and the convergence rate of the error in the $\mathbf{H}^{1}$ norm is $\mathrm{O}\left(2^{-n}\right)$, which is exactly what Theorem 4 predicts.

\section{Parallel complexity of 1D combination technique}

The number of basic operations to calculate $P_{k, l} \in V_{k, l}$ is $84 \cdot 2^{k}+83 \cdot 2^{l}+$ $115+C$, where $C$ denotes the number of basic operations involved in solving a 
TABLe 1: $\left\|P_{n, n} \mathfrak{u}-\mathfrak{u}_{\mathfrak{n}}^{\mathfrak{c}}\right\|_{\mathbf{L}^{2}}$

\begin{tabular}{rccc}
\hline $\mathrm{n}$ & $\left\|\mathrm{P}_{n, n} \mathrm{u}-\mathrm{u}_{\mathrm{n}}^{\mathrm{c}}\right\|_{\mathrm{L}^{2}}$ & ratio & ratio of $4^{-\mathrm{n} n}$ \\
\hline 1 & $5.9 \mathrm{E}-05$ & & \\
2 & $2.9 \mathrm{E}-05$ & 2.03 & 2.00 \\
3 & $1.1 \mathrm{E}-05$ & 2.66 & 2.67 \\
4 & $3.7 \mathrm{E}-06$ & 2.95 & 3.00 \\
5 & $1.2 \mathrm{E}-06$ & 3.00 & 3.20 \\
6 & $3.5 \mathrm{E}-07$ & 3.48 & 3.33 \\
7 & $1.0 \mathrm{E}-07$ & 3.52 & 3.43 \\
8 & $2.9 \mathrm{E}-08$ & 3.45 & 3.50 \\
9 & $8.0 \mathrm{E}-09$ & 3.66 & 3.55 \\
10 & $2.1 \mathrm{E}-09$ & 3.70 & 3.60 \\
11 & $5.8 \mathrm{E}-10$ & 3.72 & 3.64 \\
12 & $1.7 \mathrm{E}-10$ & 3.34 & 3.67 \\
\hline
\end{tabular}

TABLE 2: $\left\|P_{n, n} u-u_{n}^{c}\right\|_{\mathbf{H}^{1}}$

\begin{tabular}{rccc}
\hline $\mathrm{n}$ & $\left\|\mathrm{P}_{\mathrm{n}, \mathrm{n}} \mathrm{u}-\mathrm{u}_{\mathrm{n}}^{\mathrm{c}}\right\|_{\mathbf{H}^{1}}$ & ratio & ratio of $2^{-\mathrm{n}}$ \\
\hline 1 & $4.1 \mathrm{E}-04$ & & \\
2 & $2.7 \mathrm{E}-04$ & 1.48 & 2 \\
3 & $1.4 \mathrm{E}-04$ & 1.88 & 2 \\
4 & $8.5 \mathrm{E}-05$ & 1.73 & 2 \\
5 & $6.5 \mathrm{E}-05$ & 1.30 & 2 \\
6 & $2.2 \mathrm{E}-05$ & 2.85 & 2 \\
7 & $9.8 \mathrm{E}-06$ & 2.33 & 2 \\
8 & $4.8 \mathrm{E}-06$ & 2.01 & 2 \\
9 & $2.1 \mathrm{E}-06$ & 2.29 & 2 \\
10 & $1.2 \mathrm{E}-06$ & 1.73 & 2 \\
11 & $7.3 \mathrm{E}-07$ & 1.67 & 2 \\
12 & $4.0 \mathrm{E}-07$ & 1.83 & 2 \\
\hline
\end{tabular}


tridiagonal linear system. Hence, the number of basic operations to calculate $P_{n, n} \in V_{n, n}$ is $167 \cdot 2^{n}+115+C$ and the number of basic operations to calculate the $1 \mathrm{D}$ combination technique is

$$
\sum_{k+l=n}\left(84 \cdot 2^{k}+83 \cdot 2^{l}+115+C\right)+\sum_{k+l=n-1}\left(84 \cdot 2^{k}+83 \cdot 2^{l}+115+C\right) \cdot(7)
$$

Equation (7) indicates that the number of basic operations involved in the 1D combination technique is larger than the number of basic operations involved in the full grid approximation. However, from a parallel coding point of view, there are $2 n+1$ independent problems of the maximum size $84 \cdot 2^{n}+198+C$. Thus the optimal parallel complexity is $84 \cdot 2^{n}+198+C$. This reveals the best possible speedup of the $1 \mathrm{D}$ combination technique is 2 although $2 n+1$ processors are required.

To increase the best possible speedup of the $1 \mathrm{D}$ combination technique, one could initially divide the domain interval $[0,1]$ into more subintervals. This will result in variants of the 1D combination technique. Figures 1 and 2 display numerical results for the parallel implementation.

The 1D combination technique and its variants create a method to have parallel computing in one dimensional cases. The tensor product of the 1D combination technique can be used for multi-dimensional cases. For high dimensional cases, the 1D combination technique can be used together with the sparse grids technique $[2,3]$ to increase the parallelism and reduce the parallel complexity.

\section{Conclusion}

The 1D combination technique is related to Domain Decomposition and Multi-parameter extrapolation [5]. Domain Decomposition methods combine approximate solutions in different sub-domains while Combination methods 


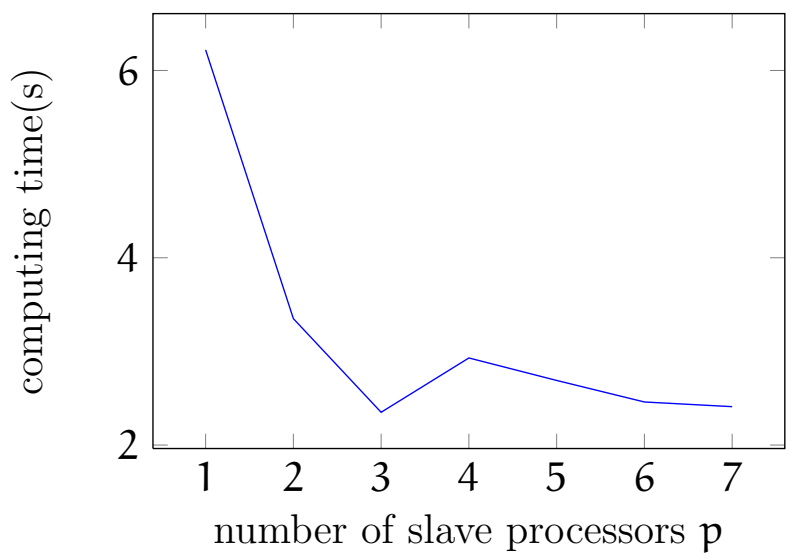

FiguRE 1: Increasing number of processors with fixed the problem size.
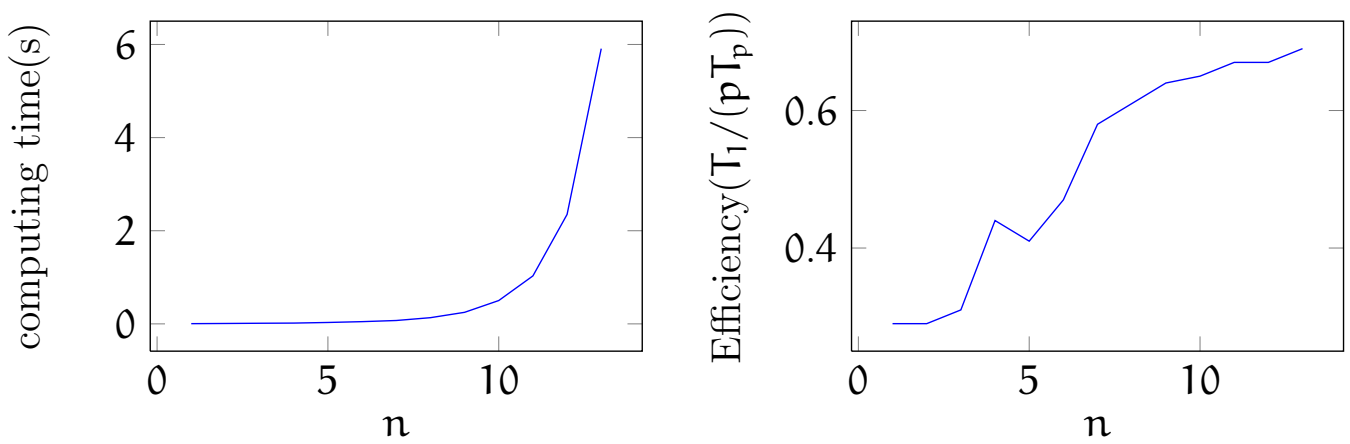

FigURE 2: Increasing the problem size with fixed number of processors. 
combine approximate solutions on different grids that are based on the entire domain.

Looking for a variant of the 1D combination technique which improves parallelism and is more accurate is left for future research. Future work will also include the extension into the $2 \mathrm{D}$ case and therefore the multidimensional case by using tensor products.

Acknowledgements I thank Markus Hegland and Nick Guoth.

\section{References}

[1] C. Zenger. Sparse grids. in Parallel Algorithms for Partial Differential Equations, Proceedings of the Sixth GSAMMSeminar, Kiel, January 19-21, 1990, W. Hackbusch, ed., Braunschweig, 1991, Vieweg-Verlag. C645

[2] M. Griebel, M. Schneider, C. Zenger. A combination technique for the solution of sparse grid problems. PdeGroen, R.Buwens(Ed.). Iterative Methods in Linear Algebra, IMACS, Elsevier, North Holland, 1992, pp.263-281. http:

//citeseer.ist.psu.edu/viewdoc/summary?doi=10.1.1.33.3530 C645, C657

[3] C. Pflaum and A. Zhou. Error analysis of the combination technique. Numerische Mathematik, 84:327-350, 1999. http:

//citeseerx.ist.psu.edu/viewdoc/summary?doi=10.1.1.54.1356 C647, C648, C657

[4] C. Pflaum. Convergence of the combination technique for second-order elliptic differential equations. SIAM J. Numer. Anal., 34(6):2431-2455, 1997. http://www. jstor.org/pss/2951959 
[5] U. Rüde and A. Zhou. Multi-parameter extrapolation methods for boundary integral equaitons. Advances in Computational Mathematics, 9:173-190, 1998.

http://www.springerlink.com/content/m311311t32345607/ C657

\section{Author address}

1. Y. Fang, Mathematical Sciences Institute, The Australian National University, ACT, Australia.

mailto:yuan.fang@anu.edu.au 